\title{
Non-Traumatic Splenic Rupture After Open Surgery for Type A Aortic Dissection: A Case Report and Literature Review
}

\author{
Xudong Guo, MD,${ }^{1}$ Xinyan Pang, $\mathrm{MD},{ }^{2}$ Zhengqin Liu, $\mathrm{MD}^{2}$ \\ ${ }^{1}$ Department of Urology, Shandong Provincial Hospital Affiliated to Shandong First Medical University, Jinan, Shandong, China; \\ ${ }^{2}$ Department of Intensive Care Unit of Cardiac Surgery, Qilu Hospital of Shandong University, Jinan, Shandong, China
}

\section{ABSTRACT}

Aortic dissection is a severe condition that involves a tear in the wall of the major artery carrying blood out of the heart (aorta). This cardiac disease has a high mortality, particularly Stanford type A, which involves the first part of the aorta. Aortic dissection is characterized by urgent onset, rapid progress, and poor outcome [Nienaber 2003; Mehta 2002]. Surgery is the most effective treatment. Although there have been remarkable recent advances in the understanding and management of various aspects of these complex surgeries, many potential complications remain.

Non-traumatic splenic rupture is a rare postoperative complication of type A aortic dissection, and there are only a few published reports discussing this complication. Splenic rupture is a life-threatening cause of intraperitoneal bleeding and often is associated with the preexisting pathology of the spleen in the absence of trauma [Renzulli 2009]. Its manifestation is characterized by abdominal pain, left shoulder pain, and even shock. Because of the history of aortic dissection, physicians initially may suspect arterial rupture. In our report, we aim to present the possibility of splenic rupture as a complication of aortic dissection surgery and the need for immediate surgical intervention.

\section{CASE REPORT}

A 60-year-old man was admitted to our cardiac surgery intensive care unit with a chief complaint of the sudden onset of severe chest and back pain. The pain started 20 hours previously when he was working, and the pain was accompanied by sweating, fatigue, and nausea. The patient had uncontrolled hypertension for more than 10 years. On admission, the patient still was suffering from intense chest pain, and physical examination revealed an elevated blood pressure of $180 / 110 \mathrm{mmHg}$, heart rate of $98 \mathrm{bpm}$, and oxygen saturation of $98 \%$ in the room air. The patient was given a general

Received August 21, 2019; received in revised form October 5, 2019; accepted February 27, 2020.

Correspondence: Zhengqin Liu, Department of Intensive Care Unit of Cardiac Surgery, Qilu Hospital of Shandong University, Finan, Shandong, China; (e-mail: robustguo@sina.com) therapy of urapidil and esmolol to reduce blood pressure and heart rate accompanied by analgesia and sedation treatment. The routine blood tests were almost normal. The ECG showed ST-segment depression in leads V1-V6. Acute aortic dissection was highly suspected, and the patient underwent emergent CT contrast-enhanced angiography, which confirmed the diagnosis of type A aortic dissection with a sizeable false lumen. The bedside ultrasound revealed severe aortic regurgitation, in which blood leaks back through the aortic valve. Based on all the tests above, there were no contraindications to the surgery. On the second day of hospitalization, surgery using a modified elephant trunk stent-graft was performed under general intratracheal anesthesia.

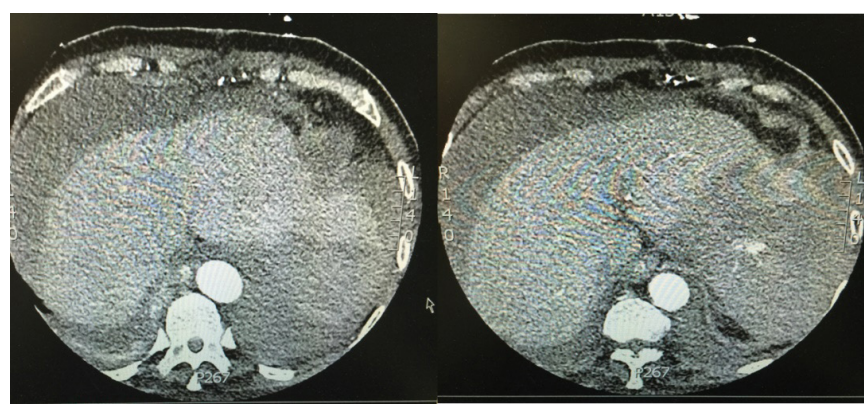

Figure 1. The CT test revealed the rupture of the spleen.

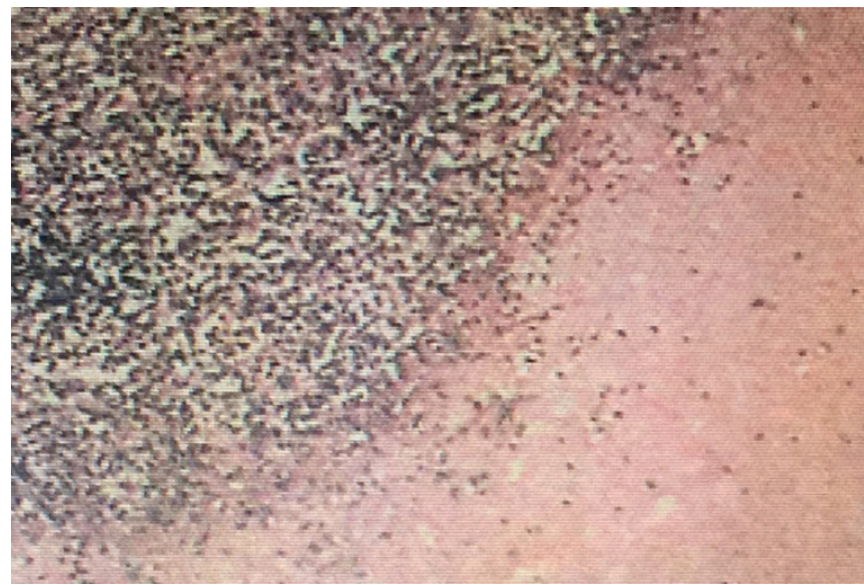

Figure 2. Microscopic examination demonstrated chronic splenic congestion accompanied by hemorrhaging. 
The patient was monitored in our intensive care unit directly after surgery, and his general condition was good. He regained consciousness 8 hours later and was extubated after 16 hours from surgery. Since the patient underwent the procedure of mechanical aortic valve replacement, he had to take the oral anticoagulant warfarin to maintain the International Normal Ratio (INR) in a range of 1.5-2.5. Other treatments were supported similarly as before the surgery, and the patient recovered smoothly.

On the eighth postoperative day, the patient complained of a sudden upper abdominal pain with progressive distension of the abdomen after coughing. After a short time, he was found to be in hypovolemic shock (BP $87 / 54 \mathrm{mmHg}$ and HR $129 \mathrm{bpm})$. There was marked pallor, and his extremities were cold and clammy. Physical examination of the abdomen revealed diffuse tenderness and board-like rigidity. The diagnostic abdominocentesis spared no clotting. Rupture of the aortic artery was first suspected, and the patient urgently was transferred to a CT-room for examination with transfusion of $20 \mathrm{mg}$ VitK1 and a large amount of liquid. The CT test revealed the rupture of the spleen (Figure 1). The patient immediately was transferred to the operating theatre, and a splenectomy was performed with the help of a general surgeon. Approximately 6L of blood was found in the abdominal cavity. The removed spleen was of normal size $10 \times 5 \mathrm{~cm}$ and friable with multiple fractures. Microscopic examination demonstrated chronic splenic congestion accompanied by hemorrhaging (Figure 2).

The patient's postoperative condition was good, and his vital signs became regular with BP 143/81mmHg and pulse of $78 \mathrm{bpm}$. On the fifth day after the operation, the abdominal drain was removed, and on the tenth day, the patient was discharged in good general condition.

\section{DISCUSSION}

In our case, the rupture of the spleen may be related to the medical treatment with the anticoagulant warfarin and the increased fragility of the spleen, due to the aortic dissection, which influenced the blood perfusion of the spleen. Under these conditions, daily movements, including severe vomiting, coughing, sneezing, or even defecation, can lead to the rupture of the spleen [Debnath 2002].

Splenic rupture is a common severe abdominal emergency usually caused by blunt abdominal trauma, but non-traumatic splenic rupture is a rarer event, only accounting for $2.9 \%-4 \%$ of cases [Jian 2015]. It was first documented in the 19th century [Weaver 2013]. Non-traumatic splenic rupture often is associated with preexisting pathological abnormalities. The etiology can be divided into 6 subgroups: infectious (i.e., malaria, mononucleosis), hematologic or non-hematologic neoplasm (i.e., leukemia, non-Hodgkin's lymphoma, and malignancies causing metastasis of spleen), inflammatory (i.e., pancreatitis, amyloidosis and immuno-rheumatologic disease), congenital or structural (pregnancy, hemangiomas, infarction, and congestive splenomegaly), iatrogenic (anticoagulants, G-CSF, and hemodialysis), and idiopathic. Aubrey-Bassler et al evaluated 613 cases of splenic rupture without risk factors or previously diagnosed disease in a systemic review. The most common associated type of splenic rupture reported in the literature was infectious $(\mathrm{N}=143)$ [Aubrey-Bassler 2012].

From the articles described before, we believed that nontraumatic splenic rupture might be causally related to severe daily activities, such as coughing, vomiting, sneezing, jumping, or straining for a bowel movement, by increasing intraabdominal pressure despite pathological abnormalities. The classic symptoms and signs of splenic rupture include left upper abdominal pain, left shoulder pain radiating from diaphragmatic irritation (Kehr's sign) [Foreman 2005; Looseley 2009], anemic appearance, left upper abdominal tenderness, hemodynamic instability, or even hypovolemic shock.

Bedside ultrasound is an effective, safe, and easily accessible examination that can reveal the location, scope, and depth of a ruptured spleen and also the degree of peritoneal hemorrhage. The accuracy of diagnosis can reach $90 \%[\mathrm{Hu}$ 2006]. However, this method is limited in that air echogenicities may be confused with the intensities. Computed tomography (CT) is a test with higher sensitivity when showing the air density in the solid organ. Furthermore, we can determine the severity of splenic rupture (grade I-V) on CT findings, according to the size of splenic laceration and associated hematoma [Becker 1994]. So if possible, given the condition of the patient, CT is preferred.

Splenectomy is the preferred treatment for most nontraumatic splenic rupture, especially with high-grade $(\mathrm{IV}-\mathrm{V})$ injuries. Low-grade (I-II) injuries conservatively may be managed. However, the clinical situation is the most important factor in guiding treatment decisions. Some patients with high-grade splenic injuries may conservatively be managed, if they are hemodynamically stable and delayed splenic rupture whose initial CT shows low-grade splenic injury may require surgery conversely.

Although non-traumatic splenic rupture is not prevalent, clinicians should have a high index of suspicion of it, particularly in patients with acute left-sided abdominal pain since its fatal outcome.

\section{CONCLUSION}

In summary, although there is nothing in the literature about the complication of atraumatic splenic rupture after type A aortic dissection, the possibility of occurrence must be considered in the case of acute abdominal pain, despite the high probability of aortic re-tearing. Especially for patients receiving anticoagulant therapy, daily movement should be kept to a proper degree to avoid severe physical force on the spleen.

\section{REFERENCES}

Aubrey-Bassler FK, Sowers N, et al. 2012. 613 cases of splenic rupture without riskfactors or previously diagnosed disease: a systematic review. BMC Emerg Med 12:11. 
Becker CD, Spring P, Glattli A, Schweizer W. 1994. Blunt splenic trauma in adults: can CT findings be used to determine the need for surgery. AJR 162(2): 343-47.

Debnath D, Valerio D. 2002. Atraumatic rupture of the spleen in adults. J R Coil Surg Edinb 47:437-445.

Foreman BH, Mackler L, Malloy ED. 2005. Can we prevent splenic rupture for patients with infectious mononucleosis? J Fam Pract 54: 547-487.

Hu Mingrong, Xu Dezheng. 2006. Spontaneous splenic rupture. J Clin Surg 7:411-412.

Liu J, Liu C, Feng Y, et al. 2015. Non-traumatic splenic rupture a report of 5 cases and review of the literatures. Chin J Hepatobiliary Surg 5:313-316.

Looseley A, Hotouras A, Nunes QM, Barlow AP. 2009. Atraumatic splenic rupture secondary to infectious mononucleosis: a case report and literature review. Grand Rounds emedicine DOI: 10.1102/1470-5206.2009.0002.

Mehta RH, Suzuki T, et a1. 2002. International Registry of Acute Aortic dissection (IRAD) Investigators Predicting death in patients with acute type A aortic dissection [J]. Circulation 105(2):200-206.

Nienaber CA, E,z,h KA. 2003. Aortic dissection: new frontiers in diagnosis and management: Part I: from etiology to diagnostic strategies [J]. Circulation 108(5):628-635.

Renzulli P, Hostettler A, Schoepfer AM, Gloor B, Candinas D. 2009. Systematic review of atraumatic splenic rupture. Br J Surg 96:1114-21.

Weaver H, Kumar V, Spencer K. 2013. Spontaneous splenic rupture: a rare life-threatening condition; Diagnosed early and managed successfully [J]. Am J Case Rep 14: 13-15. 